Draft version July 9, 2018

Preprint typeset using $\mathrm{LAT}_{\mathrm{E}} \mathrm{X}$ style emulateapj v. 12/16/11

\title{
IN DISGUISE OR OUT OF REACH: FIRST CLUES ABOUT IN SITU AND ACCRETED STARS IN THE STELLAR HALO OF THE MILKY WAY FROM GAIA DR2
}

\author{
M. Haywood and P. Di Matteo \\ GEPI, Observatoire de Paris, PSL Research University, CNRS, Sorbonne Paris Cité, 5 place Jules Janssen, 92190 Meudon, France \\ M. D. LEHNERT \\ Sorbonne Université, CNRS UMR7095, Institut dAstrophysique de Paris, 98 bis bd Arago, 75014 Paris, France \\ O. SNAITH \\ School of Physics, Korea Institute for Advanced Study, 85 Hoegiro, Dongdaemun-gu, Seoul 02455, Republic of Korea \\ S. KhOperskov AND A. Gómez \\ GEPI, Observatoire de Paris, PSL Research University, CNRS, Sorbonne Paris Cité, 5 place Jules Janssen, 92190 Meudon, France \\ Draft version July 9, 2018
}

\begin{abstract}
We investigate the nature of the double color-magnitude sequence observed in the Gaia DR2 HR diagram of stars with high transverse velocities. The stars in the reddest-color sequence are likely dominated by the dynamically-hot tail of the thick disk population. Information from Nissen \& Schuster (2010) and from the APOGEE survey suggests that stars in the blue-color sequence have elemental abundance patterns that can be explained by this population having a relatively low starformation efficiency during its formation. In dynamical and orbital spaces, such as the "Toomre diagram', the two sequences show a significant overlap, but with a tendency for stars on the blue-color sequence to dominate regions with no or retrograde rotation and high total orbital energy. In the plane defined by the maximal vertical excursion of the orbits versus their apocenters, stars of both sequences redistribute into discrete wedges. We conclude that stars which are typically assigned to the halo in the solar vicinity are actually both accreted stars lying along the blue sequence in the HR diagram, and the low rotational velocity tail of the old Galactic disk, possibly dynamically heated by past accretion events. Our results imply that a halo population formed in situ and responsible for the early chemical enrichment prior to the formation of the thick disk is yet to be robustly identified, and that what has been defined as the stars of the in situ stellar halo of the Galaxy may be in fact fossil records of its last significant merger.
\end{abstract}

Keywords: Galaxy: evolution — Galaxy: kinematics and dynamics — Galaxy: halo

\section{INTRODUCTION}

Over the past three decades, a consensus has developed that the stellar halo of the Milky Way is composed of two populations of stars - those that were born in other galaxies and accreted and those that were born in situ during the early evolution of the Milky Way (e.g. Searle \& Zinn 1978; Sommer-Larsen \& Zhen 1990; Carollo et al. 2007). Although these two components have remained challenging to characterize, we now have a widely-accepted picture whereby the in situ halo population is older, more metal-rich, dominates the stellar density within $\sim 15 \mathrm{kpc}$ of the Galactic center, and has a slightly enhanced mean rotation rate compared to the accreted halo population (e.g., Carollo et al. 2007). While a considerable amount of effort is currently being expended investigating stellar streams that are expected to fill the outer stellar halo, the inner halo population is still lacking a proper characterization, and its evolutionary connection with the thick disk is essentially unknown.

The second data release Gaia Collaboration et al. 2018a) of the European Space Agency's Gaia mission

misha.haywood@obspm.fr
(Gaia Collaboration et al. 2016) provides superb astrometric parameters, radial velocities and photometry for a large number of stars. First results have already detected a number of streams and kinematic groups (Malhan et al. 2018 Koppelman et al. 2018). Inspection of the Gaia HR diagram (hereafter HRD) of stars with high total or tangential velocities has shown two parallel color sequences (Gaia Collaboration et al.|2018b), which were attributed to stars in the thick disk and stellar halo. Here, we concentrate on understanding the origin of these two sequences and in particular the nature of the blue-color sequence. The next section describes our selection from the Gaia archive, Section 2 analyzes the sample of stars from Nissen \& Schuster (2010) specifically with respect to where stars in this sample lie in these two sequences and thus bringing insights into the characteristics of the blue-color sequence. In Section 2.3 , we present their main kinematic and orbital properties and we discuss our results and summarize our main conclusions in Section 4.

\section{CLUES FROM NISSEN \& SCHUSTER (2010)}



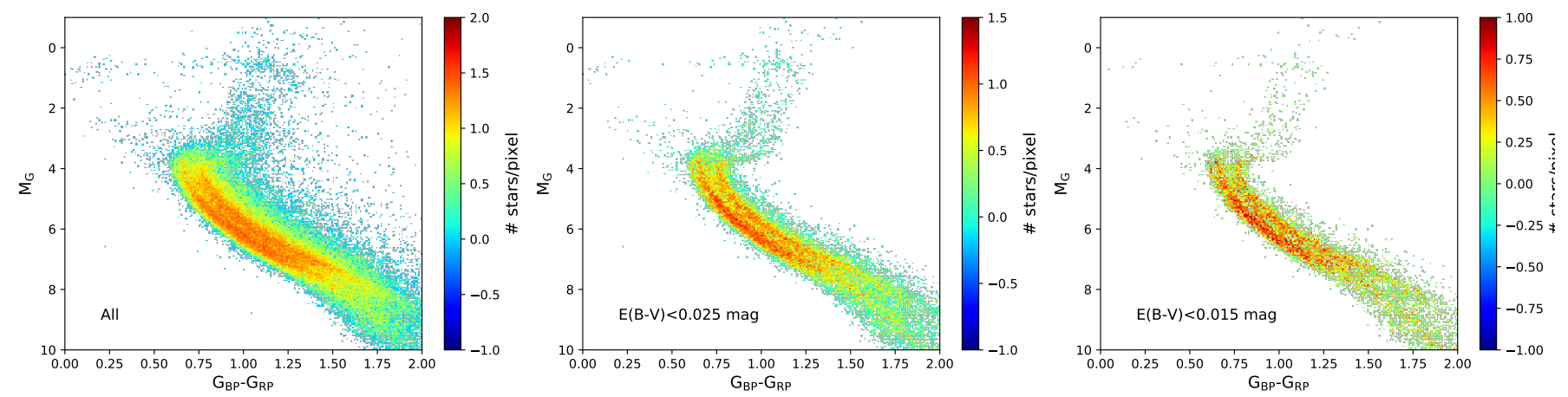

Figure 1. HRD of stars selected in Section 2.1 with different tolerances in the reddening. The two, blue- and red-color sequences, are well separated.

We select stars in the Gaia archive having a tangential velocity greater than $200 \mathrm{~km} . \mathrm{s}^{-1}$, parallax errors $\sigma_{\pi} / \pi<0.1, \pi>1$ mas, $\mathrm{G}<17$, and the filters described in Gaia Collaboration et al. (2018b). This brings us 77107 stars displayed in the HRD of Fig. 1 (left panel). This figure shows that the HRD separates in two sequences, as in Gaia Collaboration et al. (2018b), and which we designate below as the blue and the red sequence (BS and RS, respectively).

In order to clean the HRD, we select stars with various levels of tolerance on the interstellar reddening. We adopt the interstellar reddening estimates from the map of Lallement et al. (2018), which is well adapted for stars nearer than $1 \mathrm{kpc}$. Fig. 1 (middle and right panels) shows the HRD obtained by selecting stars that have levels of reddening below 0.025 and 0.015 mag, providing respectively 28210 and 12620 objects.

\subsection{Chemical abundances and HR diagram}

The sample of Nissen \& Schuster (2010) (hereafter NS) in three different metallicity intervals is overplotted to the Gaia HRD in Fig. 2, together with isochrones from the PARSEC library (Marigo et al. 2017) at $\mathrm{Z}=(0.006$, $0.0024,0.0048)$ and ages(11, 11.5, 12.5) Gyr. Note that the Gaia magnitudes were derived using the V,I magnitudes and color transformations provided by Evans et al. (2018).

This figure shows that the red and blue sequences are dominated by metal-rich $([\mathrm{Fe} / \mathrm{H}]>-0.8)$ and metal-poor $([\mathrm{Fe} / \mathrm{H}]<-1.1)$ stars (as already anticipated in Gaia Collaboration et al. 2018b). Note that the spread in metallicity in each sequence is significant, $\sim 0.6$ dex. A crossmatch between APOGEE (Majewski et al. 2017) and our Gaia sample yields 226 stars, showing that there is a marked dip in the metallicity distribution of stars at $[\mathrm{Fe} / \mathrm{H}] \sim-1.0$ (Gaia Collaboration et al. 2018b). This dip is also noticeable in the data of NS (their Fig. 1). The middle plot of Fig. 2 shows that stars with $-1.1<[\mathrm{Fe} / \mathrm{H}]<-$ 0.8 , which brackets the dip in metallicity, fall between the two sequences of the HRD. This implies that the clear separation between the two sequences is the conspicuous consequence of the dip in the metallicity distribution function (MDF). Moreover, the middle plot shows that, at similar metallicities, low- $\alpha$ stars tend to be bluer than high- $\alpha$ ones, suggesting that they must be slightly younger, supporting similar results from Schuster et al. (2012). On the contrary, the bottom panel of Fig. 2 shows that low and high- $\alpha$ stars with $[\mathrm{Fe} / \mathrm{H}]<-$
1.1 from the NS sample can both be found along the BS stars. This is surprising, because - given the tightness of the blue-color sequence - if the low and high- $\alpha$ stars at $[\mathrm{Fe} / \mathrm{H}]<-1.1$ were belonging to two different chemical evolution sequences - as they do at $-1.1<[\mathrm{Fe} / \mathrm{H}]<-0.8-$ we would expect a difference in age that would reflect in the HRD, as is seen for their more metal-rich counterparts (Fig 2, middle plot). Is it thus possible that all the stars with $[\mathrm{Fe} / \mathrm{H}]<-1.1$ - both high or low $\alpha$ stars, because the distinction becomes less obvious below this metallicity - are the same population of stars, and are not causally related to the thick disk? Could they instead be causally related to the low- $\alpha$ sequence at higher metallicities, which is clearly distinct from the thick disk?

By analyzing the $[\mathrm{Fe} / \mathrm{H}]-[\mathrm{Mg} / \mathrm{Fe}]$ distribution of APOGEE stars, Hayes et al. (2018) have shown that the separation between the high and low- $\alpha$ stars is not horizontal - it is not a separation in $\alpha$-abundances - but that high- $\alpha$ stars at $[\mathrm{Fe} / \mathrm{H}] \lesssim-1.1$ and low- $\alpha$ stars at higher metallicities form a unique chemical sequence. The findings of Hayes et al. (2018) strongly support our suggestion. Fig $3[\mid[$ shows the $[\mathrm{Fe} / \mathrm{H}]-[\alpha / \mathrm{Fe}]$ distribution of the sample of Nissen \& Schuster (2010), with the high- $\alpha$, low metallicity stars shown as blue square. Indeed, these objects are all within the BS. It is therefore reasonable to suggest that the low-metallicity $([\mathrm{Fe} / \mathrm{H}]<-1.1)$ stars of NS and the low- $\alpha$ sample at higher metallicity are forming a unique abundance sequence. The gap in the MDF at $[\mathrm{Fe} / \mathrm{H}] \sim-1$ could simply be a reflection of the transition between two populations of stars: a population whose origin needs to be determined (see below) at $[\mathrm{Fe} / \mathrm{H}]<-1$, and the thick disk above this limit. Hayes et al. (2018) also mention that the transition between the two sequences in the $[\mathrm{Fe} / \mathrm{H}]-[\alpha / \mathrm{Fe}]$ plane is more pronounced at this metallicity (see also Bonaca et al. 2017).

\subsection{Kinematics and orbits}

Of the sample of 28210 stars with $\mathrm{E}(\mathrm{B}-\mathrm{V})<0.025 \mathrm{mag}$, 1973 stars have full 3D velocity information in Gaia DR2. In all the following, we have assumed an in-plane distance of the Sun from the Galactic center $R_{\odot}=8.34 \mathrm{kpc}$ following Reid et al (2014), a height of the Sun above the

\footnotetext{
${ }^{1}$ In the first version of this article, G 81-02 was found to fall blueward to the blue sequence, due to a probably eroneous extinction estimate. P. Nissen informed us that his own estimate from uvby photometry indicate a reddening of $\mathrm{E}\left(\mathrm{G}_{\mathrm{BP}}-\mathrm{G}_{\mathrm{RP}}\right)=0.043 \mathrm{mag}$, 5 times less than ours. This seems to be much more consistent with the metallicity of this star (-0.69 dex), and has been used here.
} 

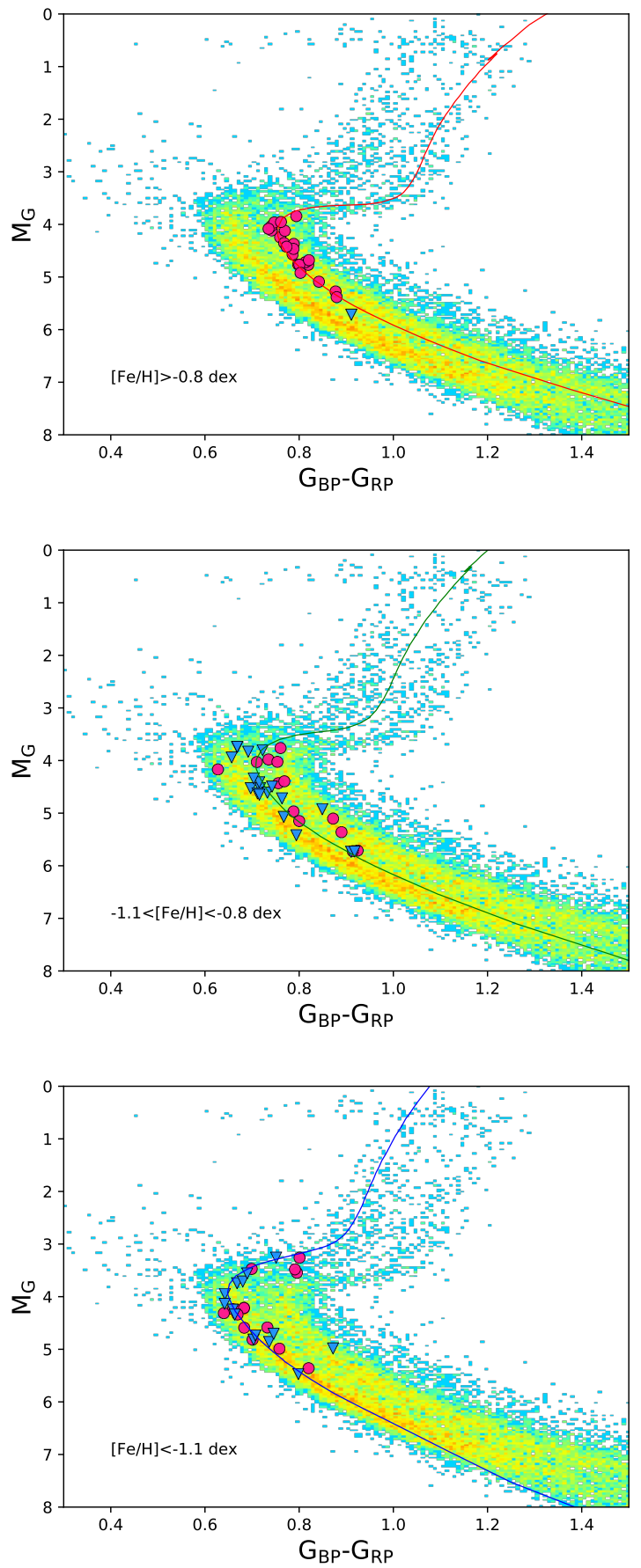

Figure 2. Overplotted on the Gaia HRD, stars from Nissen \& Schuster (2010) with: $[\mathrm{Fe} / \mathrm{H}]>-0.8$ (top panel), $-1.1<[\mathrm{Fe} / \mathrm{H}]<-0.8$ (middle panel), $[\mathrm{Fe} / \mathrm{H}]<-1.1$ (bottom panel). Thick disk and high- $\alpha$ halo stars from NS are represented with red circles, low- $\alpha$ halo stars from NS with blue triangles. The isochrones from the PARSEC library have (top to bottom): (0.006 dex, $11 \mathrm{Gyr}),(0.0048 \mathrm{dex}$, 11.5 Gyr), (0.0024 dex, 12 Gyr).

Galactic plane $z_{\odot}=27$ pc (Chen et al 2001), a velocity for the Local Standard of Rest $V_{\mathrm{LSR}}=240 \mathrm{~km} . \mathrm{s}^{-1}$ (Reid et al 2014) and a peculiar velocity of the Sun with respect to the LSR, $U_{\odot}=11.1 \mathrm{~km} . \mathrm{s}^{-1}, V_{\odot}=12.24 \mathrm{~km} . \mathrm{s}^{-1}$, $V_{\odot}=7.25 \mathrm{~km} . \mathrm{s}^{-1}$, following Schonrich al (2010).

Figure 4 show the HR diagram of the subsample of stars with 3D kinematics, and how we separate them
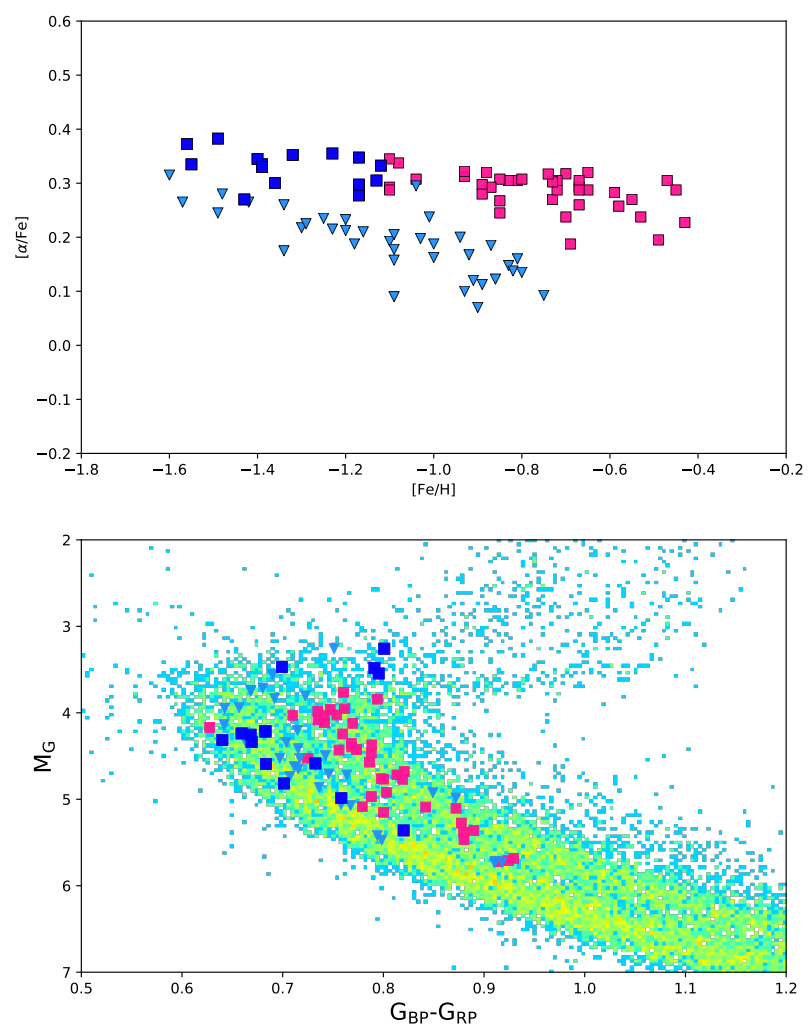

Figure 3. Top: the low- and high alpha stars from Nissen \& Schuster 2010, triangles and squares). The high- $\alpha$ stars at $[\mathrm{Fe} / \mathrm{H}]<-1.2$ plotted as blue squares may be assumed to be part of the same chemical sequence (Hayes et al. 2018). Bottom: the resulting classification is more consistent with the belonging to the two sequences in the HRD, see Fig 2

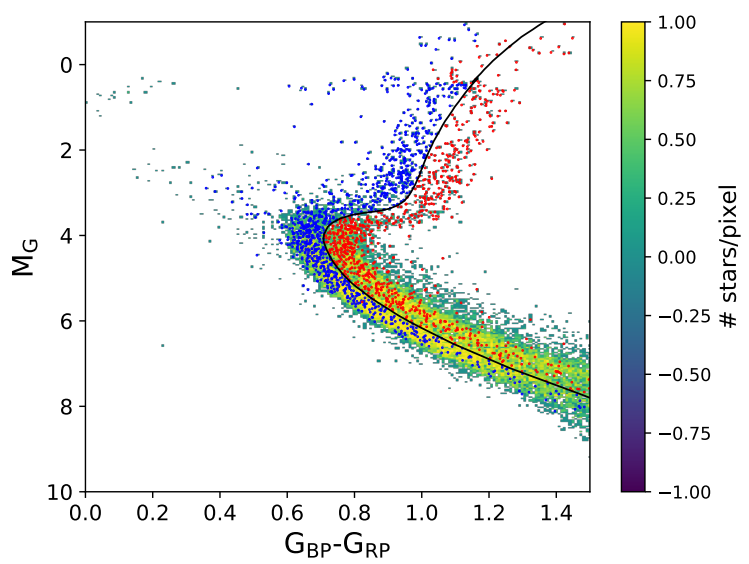

Figure 4. Gaia HR diagram of the stars with 3D kinematics in our sample. We separate our sample in two groups (red and blue sequence) according to the position of each star relative to the isochrone. The isochrone is the same as the one in Fig. 2 middle plot.

using the isochrone.

The distributions of our stars in kinematic spaces is given in Fig. 5. It shows two overlapping structures: a vertical plume which extends to high energies and was already noted in (Gaia Collaboration et al. 2018c, their Fig. 5), and is composed of stars on the blue sequence, as noted by Koppelman et al. (2018), and the high energy tail of the disk, which extends to higher rotation, and contains stars on the red sequence. The two sequences 

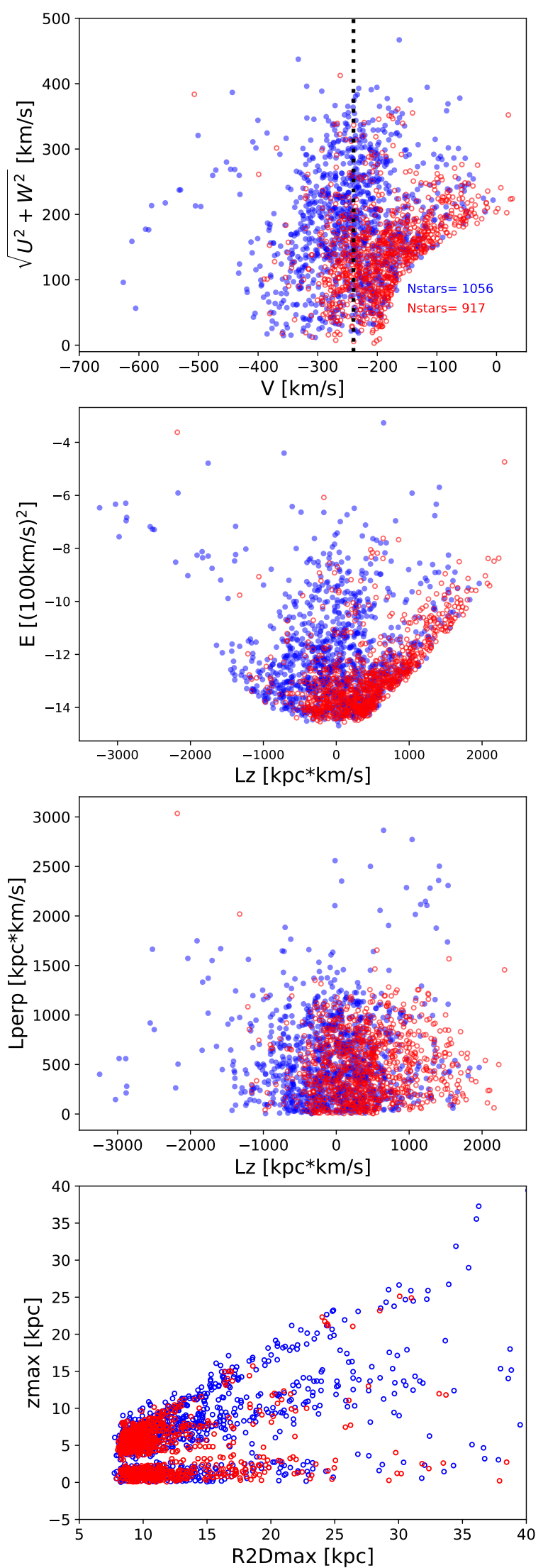

Figure 5. From top to bottom: Toomre diagram, $E-L_{z}, L_{z}-$ $L_{\text {perp }}$ and $z_{\max }-R_{2 \mathrm{D}, \max }$ planes for all stars in our sample with full 3D velocities in Gaia DR2. The dashed line in the top plot indicates $\mathrm{V}=-\mathrm{V}_{\mathrm{LSR}}$. In the bottom panel, only stars with $R_{2 \mathrm{D} \text {, max }} \lesssim 40 \mathrm{kpc}$ have been plotted. In all panels, stars on the blue sequence are shown as filled blue dots, stars on the red sequence with empty red circles. overlap in the Toomre diagram, however the majority of stars on the $\mathrm{RS}, \approx 75 \%$, shows prograde rotation and lies in the region of the diagram that is compatible with the slow rotating tail of the thick disc (Fig. 5). The remaining fraction, 1/4 of red-color sequence stars, however, has retrograde orbits, lagging the LSR by $\sim 400 \mathrm{~km}$ $\mathrm{s}^{-1}$ or less. Except for 13 stars with very significant retrograde motions, $V<-500 \mathrm{~km} / \mathrm{s}$, that we will discuss in the following, stars on the BS are distributed over the same area as the RS stars in the Toomre diagram, but in different proportions. In particular, they dominate the extended vertical plume at null or retrograde rotation, with about $65 \%$ of BS stars lying with in the region with $-350 \mathrm{~km} / \mathrm{s} \leq \mathrm{V} \leq-200 \mathrm{~km} / \mathrm{s}$, while $26 \%$ have $\mathrm{V}>-200 \mathrm{~km} / \mathrm{s}$ and the remaining fraction $\mathrm{V}<-350 \mathrm{~km} / \mathrm{s}$. Remarkably, stars of the BS with prograde orbits clearly overlap with those of RS stars.

In order to understand how these results are sensitive to the adopted classification on the red and blue sequences, we studied how the Toomre diagram changes when adopting a stricter separation. Stars have been selected respectively +0.05 redder and -0.05 bluer than the isochrone. The result is shown in Fig. 6, and shows that the effect is slightly different for the two populations: the contours of the distribution of stars on the blue sequence remain basically the same, with even the stretch of stars at strongly retrograde orbits being still present in the selection. On the contrary, several stars of the red sequence on high energy orbits have been removed, suggesting that the vertical plume, at least at the highest energies, could be almost exclusively populated by stars on the blue sequence. While a stricter separation preferentially removes stars on high energy orbits from the red sequence, we note that apart from this effect, the main characteristics of the two distributions are essentially unchanged using the two different selections.

The same main structures visible in the Toomre diagram are found also in the $E-L_{z}$ plane (Fig. 5), where the energy $E$ is the total energy of a star, defined as the sum of its kinetic and gravitational potential energies. We assume an Allen \& Santillan (1991) (hereafter AS) Galactic mass model to estimate the latter. In this plane, we note that the RS stars around $L_{z}=0$ are predominately on very bound orbits (i.e., low E). In the $L_{z}-L_{\text {perp }}$ plane, where $L_{\text {perp }}=\sqrt{L_{x}{ }^{2}+L_{y}{ }^{2}}$, there is significant overlap between the BS and RS within this space, except in two regions: (1) the region where the most extreme counter-rotating stars lie, which is composed of stars exclusively from the blue-color sequence; and (2) an over-dense region roughly centered at $\left(L_{z}, L_{\text {perp }}\right) \approx(1500,2200) \mathrm{kpc} \cdot \mathrm{km} \mathrm{s}^{-1}$, which is very close to the region occupied by the Helmi stream $\left(\left(L_{z}, L_{\text {perp }}\right) \approx\right.$ $(1000,2000) \mathrm{kpc} \cdot \mathrm{km} \mathrm{s}^{-1}$, see Helmi et al. 1999), which is also exclusively made of stars on the BS.

Finally, for all stars with full 3D velocity information, we have reconstructed their orbital parameters, by integrating their orbits over the last $6 \mathrm{Gyr}$, using four different Galaxy potentials: the axisymmetric potential of Allen \& Santillan (1991), the two axisymmetric potentials, including a thick disc component, in Pouliasis et al (2017), and the MWpotential2014 from Bovy (2015). In Fig 5, we show their distribution in the $z_{\max }-R_{2 \mathrm{D}, \max }$ 

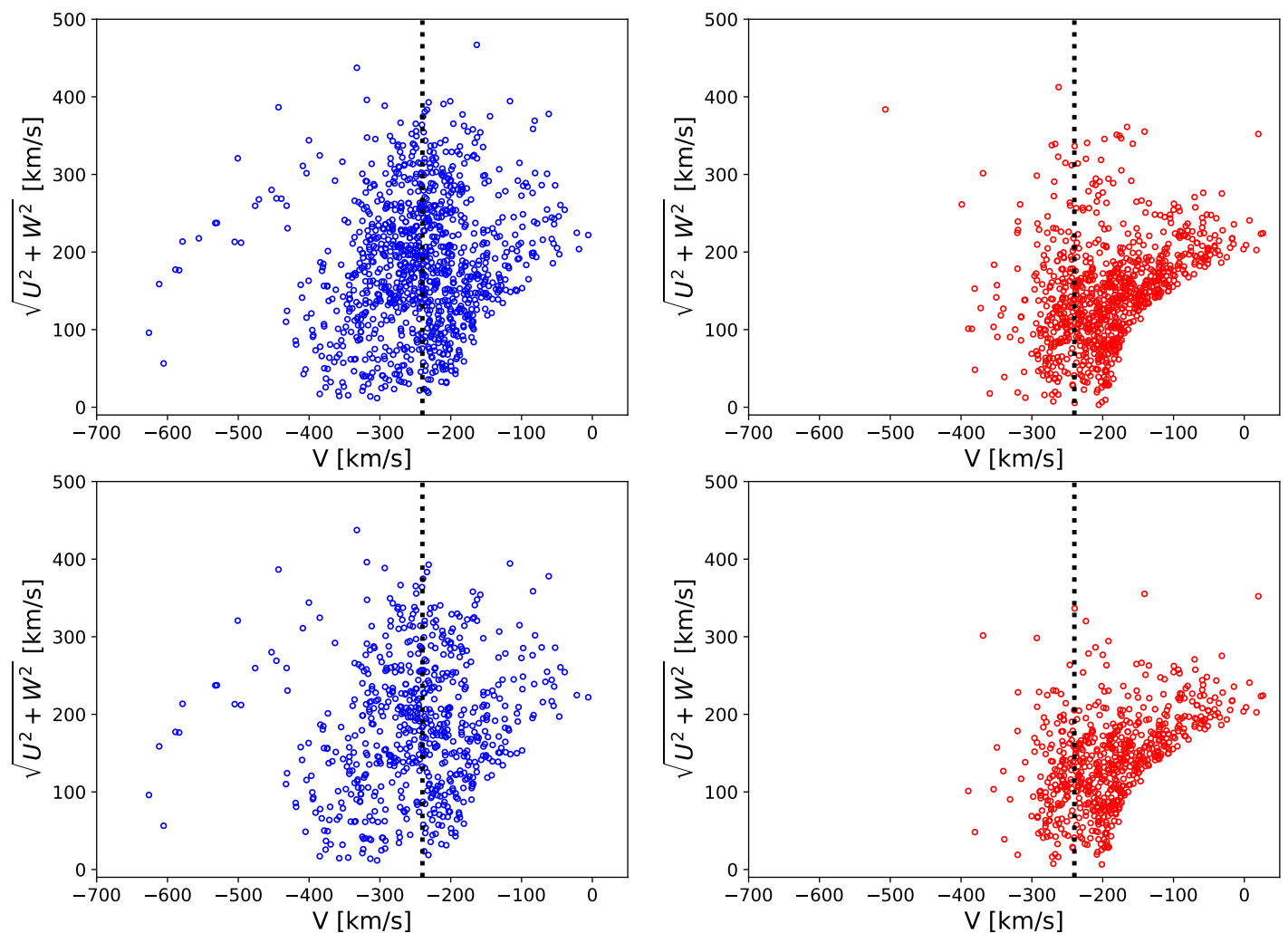

Figure 6. Top: Toomre diagram shown separately for stars on the blue and red sequences, with the same separation as in Fig. 5 Bottom: the separation is now done by selecting stars bluer by -0.05 than the isochrone, and redder by +0.05 mag for the stars in red, so the two samples are separated by $0.1 \mathrm{mag}$ in color, decreasing possible contamination between the two. See text for comment.

plane, where $z_{\max }$ is the maximum height that stars reach above or below the Galactic plane and, $R_{2 \mathrm{D}, \max }$, is the apocenter of their orbit projected on the Galactic plane. For clarity, we show in this plot only the orbital parameters that have been derived by integrating the orbits in the Allen \& Santillan (1991) potential, and we discuss below the robustness of this analysis when the other three potentials are used. Predominately, the stars on the RS have $R_{2 \mathrm{D} \text {, max }}$ within $20 \mathrm{kpc}$ of the Galactic center, while the orbits of stars on the BS can extend much further out than $20 \mathrm{kpc}$. A striking feature is that the stars in our sample are not homogeneously distributed in this plane, but define three distinct diagonal "wedges", with $z_{\max }$ increasing with $R_{2 \mathrm{D} \text {, max }}$.

Remarkably, one of these three patterns defines stars confined in a relatively thin and flattened distribution, with a lack of stars between $2 \leq z_{\max } \leq 4 \mathrm{kpc}$. This lack of stars with $z_{\max }$ in this range makes this region distinct from the rest of the sample. Ten of the thirteen stars with very significant retrograde motions, $V<-500 \mathrm{~km} \mathrm{~s}^{-1}$, are found in this thin flattened disc. Note that the presence of two groups of halo stars, a first with a flattened distribution and a second with more vertically extended orbits was already noted by Schuster et al. (2012, see their Fig. 8).

In order to assess if the structures in the (RD2max, Zmax) plane are robust to (1) the uncertainties in the parallax, proper motions and radial velocity; and (2) the uncertainties in the mass distribution in the Galaxy, we generated 10 random sets of these parameters for each star around the observed values, using the 1- $\sigma$ uncertainties given in the Gaia DR2 catalogue. The orbits were then integrated from the U,V,W velocities in four different Galactic potentials described above. From the orbital parameters generated in all four potentials, we derived the angles defined by the $\arctan (Z \max / \mathrm{R} 2 \mathrm{Dmax})$, and plot the resulting distributions in Figure 7 .

The potential of Model I in Pouliasis et al (2017) is similar to the Allen \& Santillan (1991) potential, but includes a massive thick disk, but since the overall mass distribution is fitted to the same rotation curve, the overall potential is not very different, hence the distributions of Figure 7 (top) are very similar. On the contrary, Model II in Pouliasis et al (2017) was fitted to a different rotation curve (see Pouliasis et al 2017, for details), having a more massive dark matter halo, and thus its distribution differs significantly from the previous two potentials, but is similar to the distribution obtained with the MWpotential2014 from Bovy (2015).

A common feature seen in all four distributions is the dip between $10-25^{\circ}$ (Fig. 7). It is wider in the first two potentials, but is significant in all resulting distributions. The gap between the intermediate and highest wedge is visible in only one potential, but there seems to be nothing significant in the other three potentials. We conclude that (only) the dip at $10-25^{\circ}$ is significant.

\subsection{The effect of the kinematic selection}

In this study, we selected stars having tangential velocities higher than $200 \mathrm{~km} . \mathrm{s}^{-1}$. Does the cut on tangential velocities affect our conclusions? One possible way to answer this question is to compare our results with samples that were not kinematically defined. To do so, we look at the dataset of Chiba \& Beers (2000), which has been designed to avoid kinematic bias. Fig. 8 (top) 

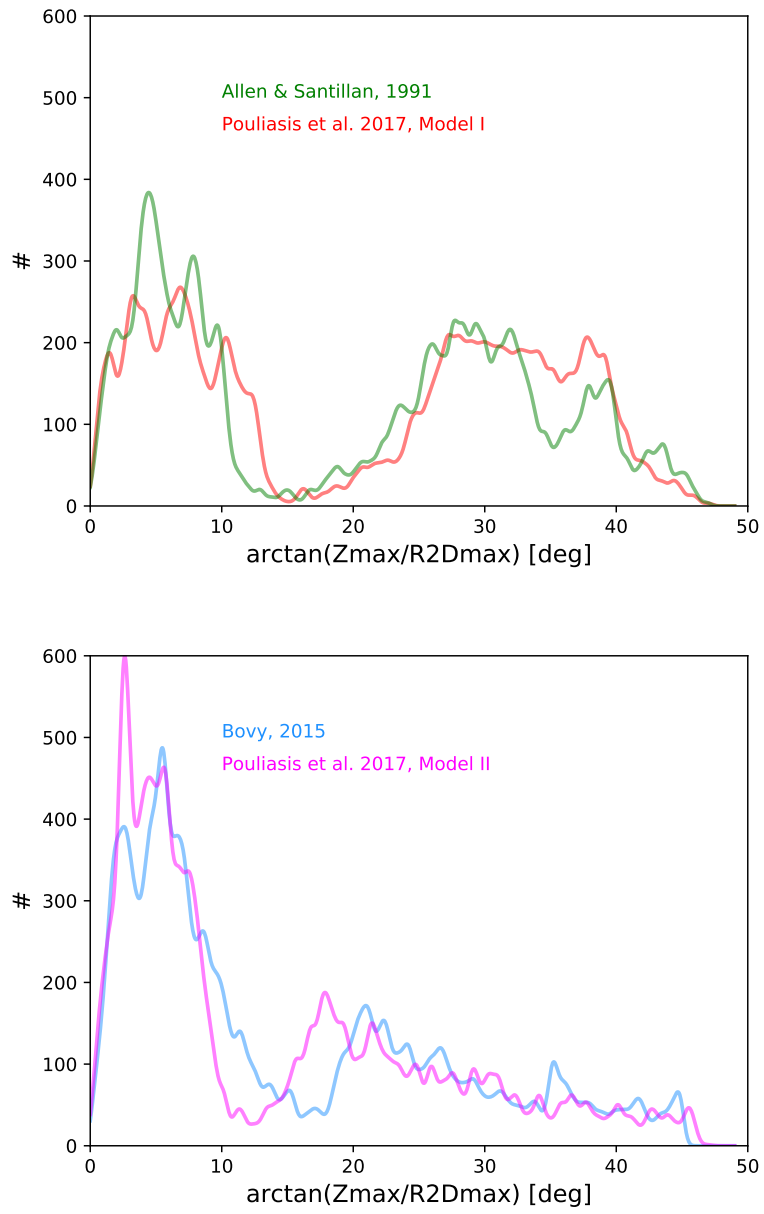

Figure 7. Distribution of angles $(\arctan (\mathrm{Zmax} / \mathrm{R} 2 \mathrm{Dmax}))$ obtained by calculating ten orbits for each star in four different Galactic potentials. The dip in the distribution (between 10 and $25^{\circ}$ ) between the lower and intermediate wedges is found in all four potentials. The dip between the intermediate and upper wedges is not.

shows the (R2Dmax,Zmax) plane adopting the orbital parameters published in Chiba \& Beers (2000), which shows that the structures were already apparent in the (Zmax,R2Dmax) plane. Figure 8 (bottom) shows the same distribution but with orbital parameters recalculated in the AS potential using the Gaia DR2 astrometric parameters. The separation between the lower and intermediate wedges is clear. The stars from Schuster et al. (2012), comprising halo stars with total velocities greater than $180 \mathrm{~km} . \mathrm{s}^{-1}$ but also thick disk stars (see Fig. 3 in Nissen \& Schuster (2010)), are also overplotted (black triangles), with most objects populating the low and intermediate wedges, with a clear separation between the two. Note that the orbital parameters of the Schuster et al. (2012) stars shown in Fig. 8 (bottom) correspond to those derived in a asymmetric barred potential (see Schuster et al. (2012) for details).

\section{THE APOGEE-GAIA SAMPLE}

In order to explore further the picture presented above, there are several limitations of our analysis so far that we can overcome. Since our sample is limited to distances where stars have $\pi>1$ mas, our sample contains
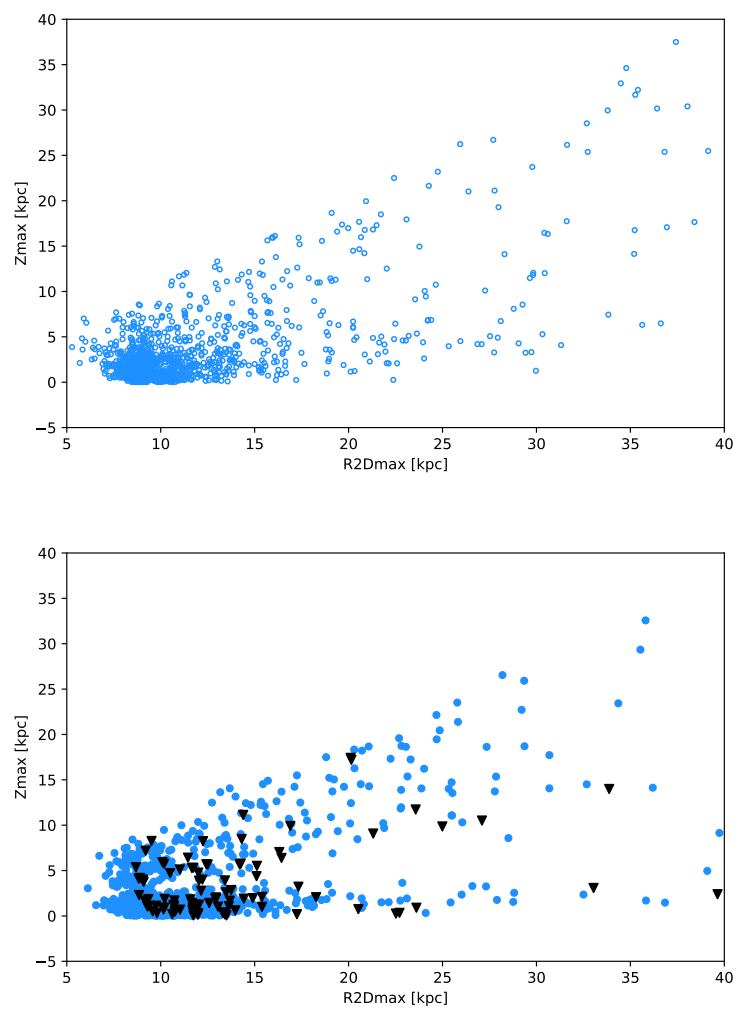

Figure 8. Top: The (R2Dmax,Zmax) distribution of stars from Chiba \& Beers (2000) with the orbital parameters determined by the authors. The same sample with the orbital parameters recalculated with the AS potential and Gaia astrometric parameters and radial velocity, together with the sample of Schuster et al. (2012) (black triangles), with their parameters derived trom orbits calculated in the barred potential.

few giants, which are the objects with the best $\mathrm{S} / \mathrm{N}$ ratio in APOGEE. Therefore, in order to include more giants, we selected in the Gaia DR2 catalogue stars having $\pi>0.3$ mas, $\sigma_{\pi} / \pi>0.1$ and $\mathrm{G}<15$. The corresponding color-magnitude diagram is shown in Fig. 9, not corrected for extinction and reddening (top) and corrected (bottom). Both sequences are well separated and densely populated and contain considerably more giants, as expected. Crossmatching this Gaia sample with APOGEE yields 950 stars with abundance estimates. Fig. 10 gives the (R2Dmax, Zmax), (E,Lz) distributions and Toomre diagram of these 950 stars, as calculated in the AS potential. As expected, the new sample follows closely the distribution of Fig. 5 ,

Based on what we learned in the previous section, we now select objects with $\mathrm{R} 2 \mathrm{Dmax}>20 \mathrm{kpc}$ on the three 'wedges' colored in blue, red and green, assuming these are the most likely to have been accreted, with the aim to see how they distribute in the $[\mathrm{Fe} / \mathrm{H}]-[\mathrm{Mg} / \mathrm{Fe}]$ plane. In the lower altitude wedge, stars are represented by two symbols according to the value of their pericenter: below or above $2 \mathrm{kpc}$ in blue or cyan respectively.

The $[\mathrm{Fe} / \mathrm{H}]-[\mathrm{Mg} / \mathrm{Fe}]$ distribution of these different subsamples is given in Fig. 11. It shows that stars with large apocenters (high energy orbits) clearly comes out as forming a low star formation efficiency sequence, typical of a relatively massive dwarf galaxy, extending from $[\mathrm{Fe} / \mathrm{H}] \sim-0.7$ to $[\mathrm{Fe} / \mathrm{H}] \sim-2.0$. 

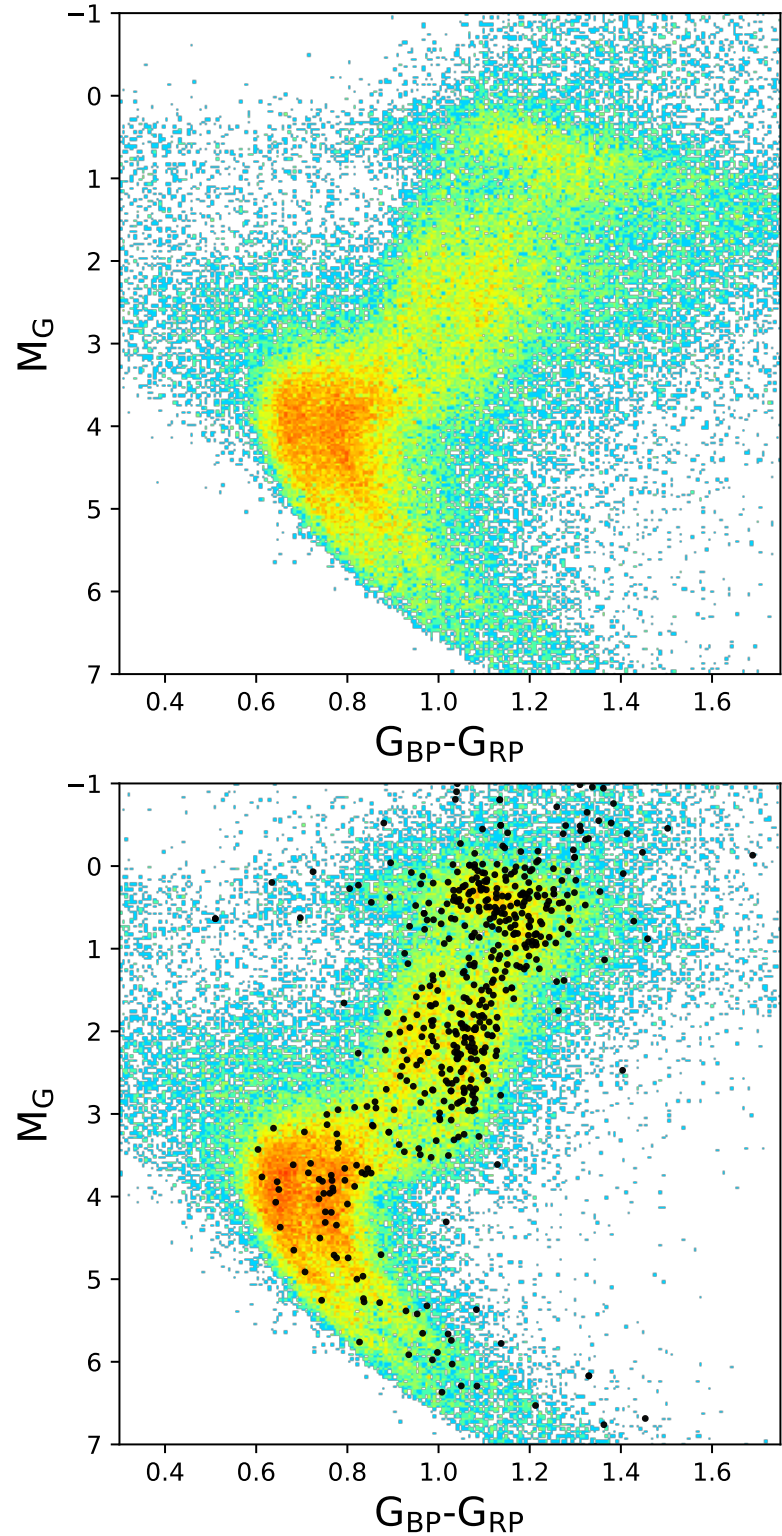

Figure 9. Gaia color-magnitude diagram for the second sample described in Section 3 not corrected for extinction (top) and corrected (bottom). The black dots on the bottom plot are the 950 stars found in the crossmatch between the Gaia sample and APOGEE.

Stars in the low altitude wedge are a mix of different origins: those colored in blue also belong to the low star formation efficiency sequence, and they differ from the others (coloured in cyan) by having pericenters smaller than $2 \mathrm{kpc}$ and which bring them near to the central regions. The objects colored in cyan have larger pericenters, typically between 3 and $7 \mathrm{kpc}$, and are mostly thick disk stars (Fig. 11). There represent the tail of this population scattered at large galactocentric distances.

Figure 10 shows as magenta dots stars that are chemically defined as thick disk objects in Fig. 11. It confirms that bona fide thick disk stars have a rotation which decreases to zero, and some are counter-rotating, up to relatively high metallicities $([\mathrm{Fe} / \mathrm{H}]>-0.5)$.

Finally, Fig. 12 shows the stars with large apocenters and those belonging to the thick disk overplotted on the color-magnitude diagrams of the Gaia sample. All stars

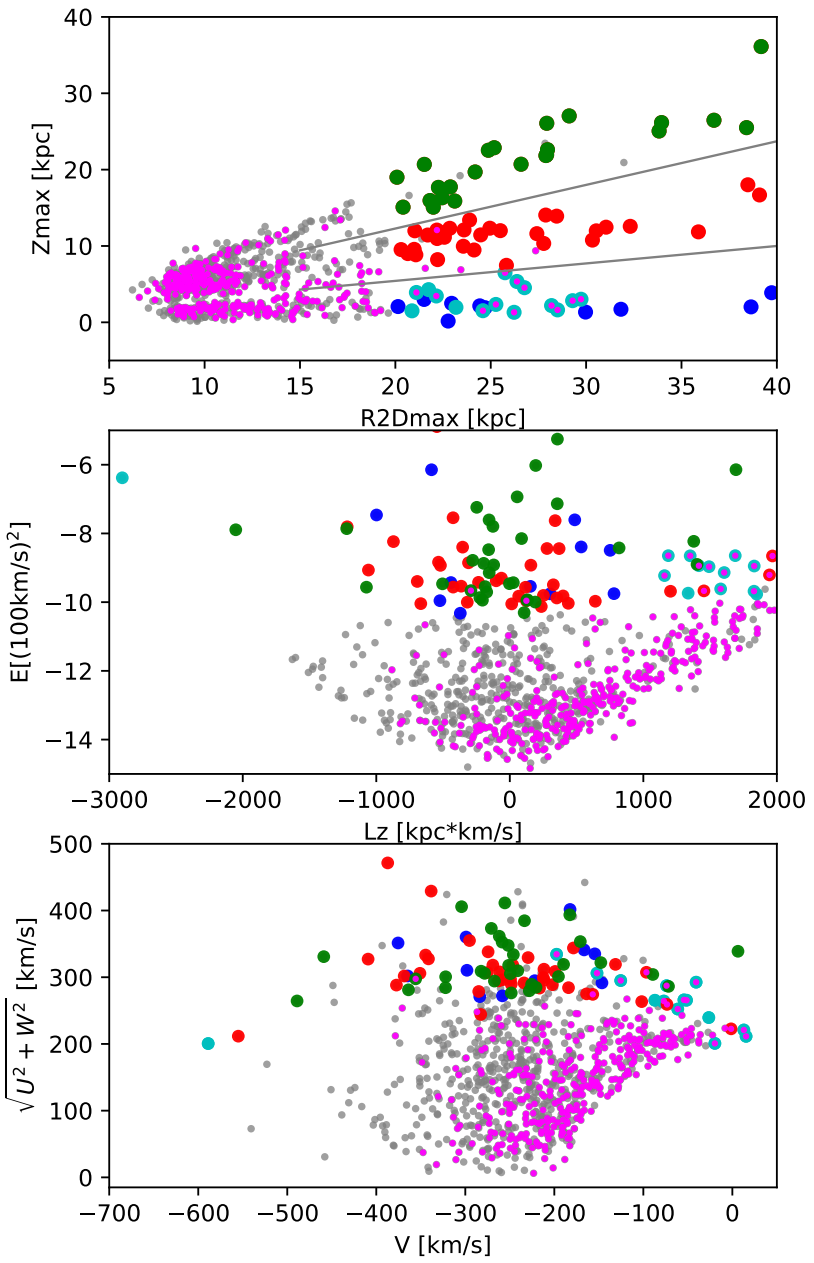

Figure 10. Top: (R2Dmax, Zmax) distribution of stars in the APOGEE-Gaia crossmatch. Stars belonging to the three wedges with R2Dmax $>20 \mathrm{kpc}$ are colored in green, red, and blue. The stars the low altitude wedge are plotted in two different colors according to the pericenter: blue for stars with R2Dmin $<2 \mathrm{kpc}$, cyan for stars with R2Dmin $>2 \mathrm{kpc}$. In magenta are the thick disk stars selected to have $[\mathrm{Fe} / \mathrm{H}]>-1$ and $[\mathrm{Mg} / \mathrm{Fe}]>0.2$ in the next figure.

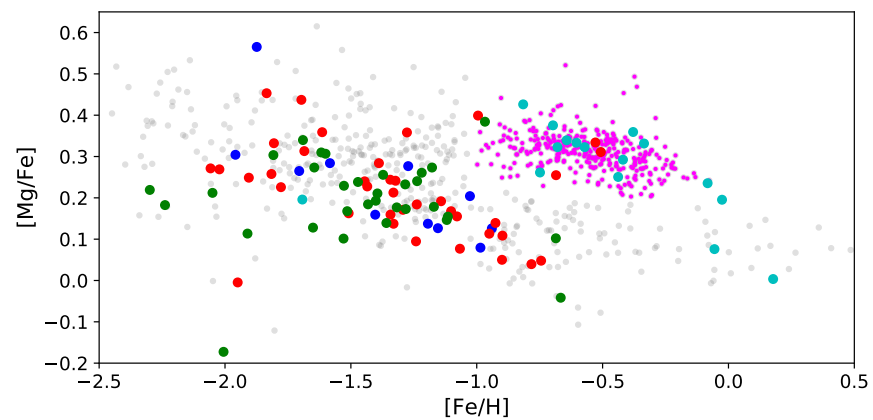

Figure 11. $[\mathrm{Fe} / \mathrm{H}]-[\mathrm{Mg} / \mathrm{Fe}]$ distribution of stars of the APOGEEGaia sample. The stars selected on the basis of their R2Dmax (blue, red, green) are the same as in Fig. 10 Thick disk stars are represented by magenta points. 


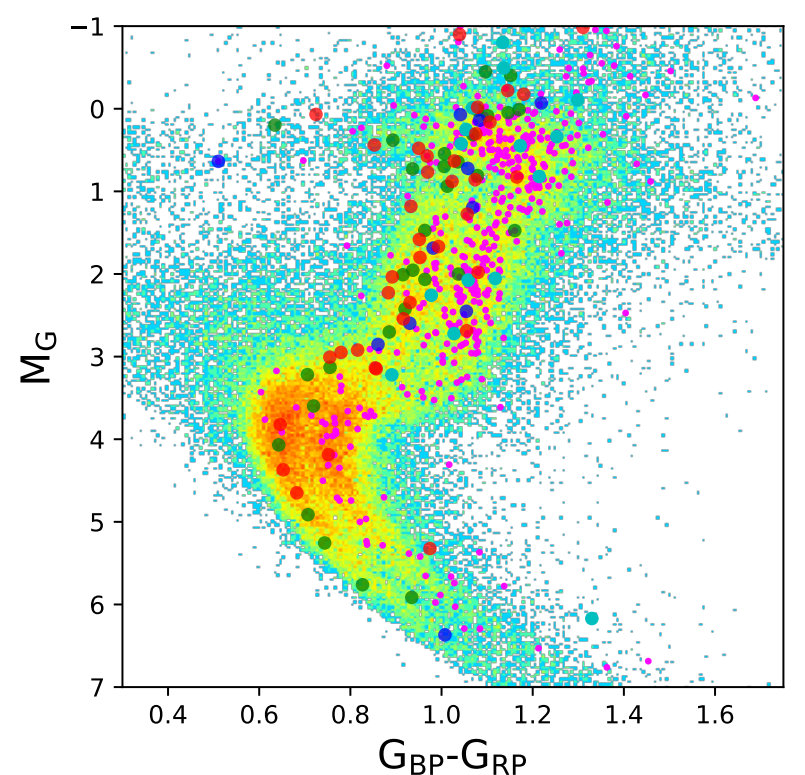

Figure 12. Gaia colour-magnitude diagram for the APOGEE stars selected as described in Sect. 3 Symbols are the same as in Fig. 10 Magnitudes have been corrected for extinction.

have been corrected for extinction. The figure confirms the previous analysis by showing that the blue sequence is dominated by stars that are on the accreted sequence, while the red sequence is dominated by thick disk objects, including those which have large apocenters but clearly belong to the chemically defined thick disk population.

\section{DISCUSSION AND CONCLUSIONS}

Our results come in the context of a long history of halo population studies. The dichotomy observed in the Gaia HRD has been known for several decades as due to the halo and thick disk. Gilmore et al. (1985) found that star counts at the pole are dominated by two populations with main-sequence turn-offs dominated by stars at $[\mathrm{Fe} / \mathrm{H}]-1.5$, and the other at $[\mathrm{Fe} / \mathrm{H}]=-0.7$. This is also what was determined in the analysis of Sloan data (Ivezić et al. 2008). More recently, Jofré \& Weiss (2011) studied the main sequence turn-off of old populations using SDSS data and determined the age of the inner Galactic halo. The flattened distribution found here, which reflects a strongly anisotropic velocity distribution, is reminiscent of the findings of Sommer-Larsen \& Zhen (1990) and echoes the analysis of Chiba \& Beers (2000). After their results, the Galactic halo sampled at the solar vicinity has been seen as an inner, flattened and in situ counterpart of a more extended, spherical, and accreted halo, a view that has been supported by other, more recent studies (see Carollo et al. 2007, 2010, Beers et al. 2012), but it must be noted that already Brook et al. (2003) proposed that eccentric stars in the sample of Chiba \& Beers (2000) could have been the result of an accretion. The main result of our study is that the inner halo is probably mainly composed of accreted material left by the last significant merger in our Galaxy.

Our results confirm that the red sequence must be dominated by thick disk stars with metallicities between -0.4 and -1 , as found in Gaia Collaboration et al. (2018b). The majority of RS stars are on prograde orbits. However, an important, new result is that a nonnegligible fraction of RS stars are on retrograde orbits.
This can be at least partially attributed, in figure 5 to an imperfect separation of stars of the BS and RS in the HRD - that is, part of the RS stars with very low or retrograde tangential velocities may be in fact BS stars which have been incorrectly assigned to the RS. Importantly, the analysis of the APOGEE sample clearly shows that some stars defined chemically as thick disk objects have retrograde orbits. It is known that in situ stellar disks, dynamically heated by one or several satellite accretion events, will also have counter-rotating stars (see Qu et al. 2011; Jean-Baptiste et al. 2017). Hence, finding some non-rotating or counter-rotating stars among an in situ old disk population is not surprising. Although they have chemical characteristics typical of the thick disk, RS stars have the kinematics of halo stars (see also Bonaca et al. 2017; Posti et al. 2017). The most likely origin of the red-color sequence is thus related to the old Galactic disk, partially heated during some past accretion event(s).

Gaia Collaboration et al. (2018b) suggest that the bluecolor sequence may be related to the in-situ halo. We find here that this sequence is most probably dominated by accreted objects, as also suggested by Koppelman et al. (2018).

Low metallicity, $[\mathrm{Fe} / \mathrm{H}]<-1.1$, high- $\alpha$ stars from Nissen \& Schuster (2010) and from the APOGEE sample studied here are indeed on the BS. Our analysis shows that also low-metallicity, low- $\alpha$ stars are on this sequence $(\S 2)$. Based on the fact that they belong to the same narrow sequence in the HRD and on the results of Hayes et al. (2018), we conjecture that the high- $\alpha$ stars with $[\mathrm{Fe} / \mathrm{H}]<-1.1$ could be causally connected to the higher metallicity, low- $\alpha$ stars forming a unique chemical evolution sequence. This is confirmed by the chemical characteristics of the stars with the highest orbital energies in our APOGEE sample: these objects form a sequences which extends from about $[\mathrm{Fe} / \mathrm{H}]=-0.5$ to at least -2.0 and from low to high- $\alpha$ abundance. Most of these objects are on the blue sequence in the Gaia HRD.

If these stars are on the same evolutionary sequence, they must have been formed in an environment characterized by a low star formation efficiency (i.e. long star formation time-scale), much lower than that of the thick disk. For this sequence, Supernovae-Ia must have started enriching the interstellar medium in Fe at lower metallicities compared to what is observed for thick disk stars of the Milky Way. The low- $\alpha$ stars in the sample of Nissen \& Schuster (2010) would then only represent the 'tip of the iceberg' of the much larger population blue-color sequence stars studied by us and APOGEE (Hayes et al. 2018). The most natural origin of the blue-color sequence is that it is dominated by stars accreted from a satellite.

The stars that most likely were accreted dominate the distribution of (Zmax,Rmax) at the largest apocenters, but it is difficult to determine the fraction they represent at lower R2Dmax. Clearly, the low- $\alpha$ sequence is not only populated by objects having large apocenter. Interestingly, the sample at metallicities lower than -2 have more limited excursions in $\mathrm{R}$ and $\mathrm{Z}$, with typical orbits being limited to within 15 and $10 \mathrm{kpc}$. This pecularity has also been noted by Schuster et al. (2012).

What may be the origin of these accreted stars? Belokurov et al. (2018) find a strong orbital anisotropy for stars with metallicities above -1.7 and low anisotropy for stars below this limit. They suggest that the major- 
ity of the halo stars within $30 \mathrm{kpc}$ are the remnant of a massive satellite accreted during the formation of the Galactic disk between about 8 and 11 Gyr ago. The analysis of Gaia DR2 data may support this scenario for several reasons: (1) BS stars seem to constitute a significant fraction of all stars with high transverse velocities; (2) we show that they could be on a chemical evolutionary track that is less $\alpha$-enriched than disk stars at the same metallicity and thus are compatible with an accreted population; (3) the kinematic properties of this BS - in particular, the high fraction of stars in retrograde orbits, a fraction of which are confined within a flattened and extended disk - and more generally the discrete wedges in the $z_{\max }-R_{2 \mathrm{D} \text {, max }}$ plane - are all reminiscent of some impulsive heating of the early Galactic disk related to some accretion event(s). In this respect, the gap in the distribution of $z_{\max }$ values may mark the transition from an early phase of significant stellar accretion in the Galaxy to a more quiescent phase.

Can all stars in the blue-color sequence be attributed to a unique satellite accretion event, or is it possible that several satellites contributed to make it? From the tightness of the BS in the HRD, it is difficult to conceive that this sequence consists of populations formed in several satellite galaxies, unless their chemical and age properties at the time of their accretion were remarkably similar. Also the kinematics of stars in this sequence may be compatible with a unique - and relatively massive merger. Fig. C.3 in Jean-Baptiste et al. (2017), for example, shows that among several satellites accreted onto a Milky Way-type galaxy, stars originating in one of them (satellite \#3 in that plot) are distributed in the $E-L_{z}$ space in a way qualitatively similar to the BS stars we have observed, with stars having both prograde and retrograde orbits, and a significant plume of stars with $L_{z}$ centered around $L_{z}=0$, and extending vertically to high energies. These simulations support the notion that the blue-color sequence is (at least partially) the remnant of a significant accretion event in the early history of the Milky Way. Note that Nissen \& Schuster (2010); Schuster et al. (2012) have evoked the possibility that the low- $\alpha$ stars in their work may originate from $\omega$ Cen, and at this stage we cannot reject or confirm this suggestion for the origin of the blue sequence. Koppelman et al. (2018) also note that previous studies have associated this region to possible debris from $\omega$ Cen. However, we note that $\omega$ Cen seems to have a peculiar barium abundance (Majewski et al. 2012) which appears not compatible with that of the low- $\alpha$ stars discussed here (see Nissen \& Schuster 2011).

While our work represents only a first exploration of the stellar halo in Gaia DR2, it is clear that this mission, together with large spectroscopic surveys, is reshaping the boundaries that we had for decades assigned to the various stellar populations of the Milky Way. Our results suggest that what has been defined as the stars of the in situ stellar halo of the Galaxy may be in fact fossil records of its last significant merger. Stars kinematically defined to belong to the inner halo comprise this possible accreted population and a sizable fraction of more metal-rich, $[\mathrm{Fe} / \mathrm{H}] \gtrsim-1$, stars that are possibly the vestiges of the early disk of the Galaxy after it was heated by one or more merger events. But where is the parent population of the thick disk? It is possible that this progenitor, the in situ, chemically-defined halo is lurking in the blue sequence, but is under-represented in the volume probed by our study? So the question remains, is the in situ halo stellar population expected in galaxy evolution models disguised among the blue-color sequence or is it still beyond our reach?

Note: A month after this article in its first version was submitted to this Journal and posted on arXiv, Helmi et al. posted an article which confirms some of our results.

We would like to thank Poul Nissen and Timothy Beers for valuable comments, the referee for several suggestions which improved this work, and David Katz and Nicolas Leclerc for helping with the use of the Gaia Archive. The Agence Nationale de la Recherche (ANR) is acknowledged for its financial support through the MOD4Gaia project (ANR-15-CE31-0007, P. I.: P. Di Matteo), also providing the postdoctoral grant for Sergey Khoperskov. This work has made use of data from the European Space Agency (ESA) mission Gaia (https://www.cosmos.esa.int/gaia), processed by the Gaia Data Processing and Analysis Consortium (DPAC, https://www.cosmos.esa.int/web/gaia/dpac/consortium). Funding for the DPAC has been provided by national institutions, in particular the institutions participating in the Gaia Multilateral Agreement. This research has made use of the SIMBAD database, operated at CDS, Strasbourg, France. Funding for the Sloan Digital Sky Survey IV has been provided by the Alfred P. Sloan Foundation, the U.S. Department of Energy Office of Science, and the Participating Institutions. SDSS-IV acknowledges support and resources from the Center for High-Performance Computing at the University of Utah. The SDSS web site is www.sdss.org. SDSS-IV is managed by the Astrophysical Research Consortium for the Participating Institutions of the SDSS Collaboration including the Brazilian Participation Group, the Carnegie Institution for Science, Carnegie Mellon University, the Chilean Participation Group, the French Participation Group, Harvard-Smithsonian Center for Astrophysics, Instituto de Astrofísica de Canarias, The Johns Hopkins University, Kavli Institute for the Physics and Mathematics of the Universe (IPMU) / University of Tokyo, Lawrence Berkeley National Laboratory, Leibniz Institut für Astrophysik Potsdam (AIP), Max-Planck-Institut für Astronomie (MPIA Heidelberg), Max-Planck-Institut für Astrophysik (MPA Garching), Max-Planck-Institut für Extraterrestrische Physik (MPE), National Astronomical Observatories of China, New Mexico State University, New York University, University of Notre Dame, Observatário Nacional / MCTI, The Ohio State University, Pennsylvania State University, Shanghai Astronomical Observatory, United Kingdom Participation Group, Universidad Nacional Autónoma de México, University of Arizona, University of Colorado Boulder, University of Oxford, University of Portsmouth, University of Utah, University of Virginia, University of Washington, University of Wisconsin, Vanderbilt University, and Yale University.

\section{REFERENCES}

Allen, C.\& Santillan, A., 1991,RMxAA, 22, 255

Beers, T. C., Carollo, D., Ivezić, Ž., et al. 2012, ApJ, 746, 34 
Belokurov, V., Erkal, D., Evans, N. W., Koposov, S. E., \& Deason, A. J. 2018, arXiv:1802.03414

Bonaca, A., Conroy, C., Wetzel, A., Hopkins, P. F., \& Kereš, D. 2017, ApJ, 845, 101

Bovy, J. 2015, ApJS, 216, 29

Brook, C. B., Kawata, D., Gibson, B. K., \& Flynn, C. 2003, ApJ, 585, L125

Carollo, D., Beers, T. C., Lee, Y. S., et al. 2007, Nature, 450, 1020

Carollo, D., Beers, T. C., Chiba, M., et al. 2010, ApJ, 712, 692

Chen, B., Stoughton, C., Smith, J. A., et al. 2001, ApJ, 553, 184

Chiba, M., \& Beers, T. C. 2000, AJ, 119, 2843

Evans, D. W., Riello, M., De Angeli, F., et al. 2018, arXiv:1804.09368

Gaia Collaboration, Prusti, T., de Bruijne, J. H. J., et al. 2016, A\&A, 595, A1

Gaia Collaboration, Brown, A. G. A., Vallenari, A., et al. 2018a, arXiv:1804.09365

Gaia Collaboration, Babusiaux, C., van Leeuwen, F., et al. 2018b, arXiv: 1804.09378

Gaia Collaboration, Katz, D., Antoja, T., et al. 2018c, arXiv:1804.09380

Gilmore, G., Reid, N., \& Hewett, P. 1985, MNRAS, 213, 257

Hayes, C. R., Majewski, S. R., Shetrone, M., et al. 2018, ApJ, 852,49

Haywood, M., Di Matteo, P., Lehnert, M., Katz, D., \& Gómez, A. 2013, A\&A, 560, A109

Haywood, M., Di Matteo, P., Snaith, O., \& Calamida, A. 2016, A\&A, 593, A82
Helmi, A., White, S. D. M., de Zeeuw, P. T., Zhao, H. 1999 Nature, 402, 53

Ivezić, Ž., Sesar, B., Jurić, M., et al. 2008, ApJ, 684, 287

Jean-Baptiste, I., Di Matteo, P., Haywood, M., et al. 2017, A\&A, 604, A106

Jofré, P., \& Weiss, A. 2011, A\&A, 533, A59

Koppelman, H. H., Helmi, A., \& Veljanoski, J. 2018, arXiv:1804.11347

Lallement, R., Capitanio, L., Ruiz-Dern, L., et al. 2018, arXiv:1804.06060

Majewski, S. R., Nidever, D. L., Smith, V. V., et al. 2012, ApJ, 747, L37

Majewski, S. R., Schiavon, R. P., Frinchaboy, P. M., et al. 2017, AJ, 154,94

Malhan, K., Ibata, R. A., \& Martin, N. F. 2018, arXiv:1804.11339

Marigo, P., Girardi, L., Bressan, A., et al. 2017, ApJ, 835, 77

Nissen, P. E., \& Schuster, W. J. 2010, A\&A, 511, L10

Nissen, P. E., \& Schuster, W. J. 2011, A\&A, 530, A15

Posti, L., Helmi, A., Veljanoski, J., \& Breddels, M. 2017, arXiv:1711.04766

Pouliasis, E., Di Matteo, P., Haywood, M. 2017, A\&A, 598, 66

Qu, Y., Di Matteo, P., Lehnert, M. D., \& van Driel, W. 2011, A\&A, 530, A10

Reid, M. J., Menten, K. M., Brunthaler, A., et al. 2014, ApJ, 783, 130

Schönrich, R., Binney, J., \& Dehnen, W. 2010, MNRAS, 403, 1829

Schuster, W. J., Moreno, E., Nissen, P. E., \& Pichardo, B. 2012 A\&A, 538, A21

Searle, L., \& Zinn, R. 1978, ApJ, 225, 357

Sommer-Larsen, J., \& Zhen, C. 1990, MNRAS, 242, 10 